\title{
Business Silos INTEgRATION USING SERVICE ORIENTED ARCHITECTURE
}

\author{
Rajashri Hiremath ${ }^{1}$ and Dr R Selvarani ${ }^{2}$ \\ ${ }^{1}$ Department of Computer Engineering, MSRIT, VTU, Bangalore, Karnataka, India \\ rajashrihiremathegmail.com \\ ${ }^{2}$ Department of Computer Engineering, MSRIT, VTU, Bangalore, Karnataka, India \\ selvarani.riic@gmail.com
}

\begin{abstract}
Agile integration satisfies the business agility and provides solutions for maintaining business changes and ensures that the enterprise survives in the current competition. Any business should be robust enough to respond to end user request. Existing traditional enterprise applications are in-capable of integrating with different business silos lacks to improve the business agility. To address this issue, this paper is about the agile integration of different business silos using "Service Oriented Architecture" and its core technology enables the business enterprise systems flexible, loosely coupled and improves agility. Enterprise business systems have to adopt Service Oriented Architecture (SOA) as it promises to help them respond more rapidly to changing business requirements by composing new solutions from existing business services. Here the definition of SOA, its layers and the core technologies have been discussed.
\end{abstract}

\section{KEYWORDS}

Service Oriented Architecture, Enterprise Service Bus, Business Process Execution Language, Business Rules Engine, Web Service Description Language, Quality of Service

\section{INTRODUCTION}

Current business systems are developed monolithically, which is difficult to integrate with other business systems and lack in co-ordination. Redundant information is resided in each of the monolithic systems and consolidation of this information from these systems is awful. Along with the rapid, worldwide development of information technology, information systems for all purposes is needed everywhere. The existing business systems have failed to meet the rapid development changes required. Also these systems possibly will not be able to adapt in intensely changing market and correspondingly changing business. A system needs to be built, which provides loose coupling, platform independence, and language independent architecture, which improves agility and act in response to the speed of a business enterprise.

To overcome this problem, SOA provides an agile based architecture towards integrating these monolithic systems, which address the technology agility to achieve the business agility by operational efficiency, cost and time-to-market and provide a unified view of information across silos.

DOI : 10.5121/ijcsea.2012.2206 


\section{BACKGROUND}

In SOA, the services and workflows have a close relationship. A number of services can constitute workflows and the service itself can be achieved based on workflows. Because of dynamic changes of the organization and service, the distributed computing method is quite different with the traditional workflow management model. Also, [4] the architecture of SOA embodies the characteristics of distributed computing. So in order to resolve the service oriented workflow management system across different organizations, a system based [13] on the structure of a service-oriented workflow management system in a number of services and the workflow management, and their communications standards can be seen. [2] Major distributed systems, such as educational system, are difficult to develop due to their complex and decentralized nature. SOA is a new form of distributed software architecture. [11] It facilitates the development of such systems by supporting modular design, application integration interoperation and software reuse. With open standards, such as XML, SOAP, WSDL and UDDI, It also supports interoperability between services operating on different platforms and between applications implemented in different programming languages. Maintainability [2] of educational services is easier than before, because educational services are divided with respect to category and functions. [7] It can be used with GIS applications too. In which it consists of a reference model, conceptual model, and a design method. Reference model shows roadmap of any future architectural and design efforts. Conceptual model introduces a conceptual service oriented solution for reference model. Finally, service oriented design method leads organizations to reach the solution through specific process. As a result, it provides general software architecture, which covers both real enterprise requirements and GIS concerns. It is an agile model with proper patterns, which increases interoperability and reusability across the enterprise. Also the model can adopt itself with any organizations and can be customized to any specific GIS applications. [7]

There are various frameworks developed with SOA for different enterprises. Few of the frameworks developed are as follows. Service-oriented architectures support distributed heterogeneous environments, where business transactions occur among loosely connected services. Ensuring a secure infrastructure for this environment is challenging. [1] There are various approaches to addressing information security, each with its own set of benefits and difficulties. Additionally, organizations can adopt vendor-based information security frameworks to assist them in implementing adequate information security controls. Unfortunately, there is no standard information security framework, which has been adopted for service-oriented architectures. Information security components for a service-oriented architecture environment are proposed based on the analysis of information security challenges faced by service-oriented architectures. [1, 16] SOA has created opportunities to improve agility and speeds in aligning business needs with information technology infrastructure. Most of the organizations in the process of applying this technology encounter with challenges and may have failure despite spending a lot of energy and huge investments. Hence they need to implement a service oriented framework for SOA governance maturity and to aim at the current status of governance, determine their requirements and then offer a suitable [15] model for it.

Three dimensions identify for the framework: maturity levels, governance maturity levels and adoption domains. Through a good framework, organization can recognize its current situation better and determine its future status easier. $[5,14]$ It is an architectural pattern providing agility to align technical solutions to modular business services that are decoupled from service consumers. Service capabilities such as interface options, quality of service, throughput, security and other constraints are described in the Service Level Agreement that would typically be published in the service registry (UDDI) for use by customers and/or mediation mechanisms. For mobile data streaming [10] applications, problems arise when a service provider's SLA attributes 
cannot be mapped one-to-one to the service consumers (i.e. $150 \mathrm{MB} / \mathrm{sec}$ video stream service provider to $5 \mathrm{MB} / \mathrm{sec}$ data consumer). Hence a generic framework prototype[9, 17] for managing and disseminating streaming data within a SOA environment as an alternative to custom service implementations based upon specific consumers or data types can be obtained. Based on that framework, a set of services were implemented to demonstrate the flexibility of streaming data framework within SOA environment. A prototype comprising a set of services that can control and disseminate data streaming within a generic framework that is language and encoding independent could be implemented. Hence unnecessary or unwanted network traffic that would otherwise tax the bandwidth, energy, and processing capacities of mobile devices could be minimized [3].

IT $[6,12]$ solutions and services are being developed, debugged, deployed and delivered from clouds to be conveniently consumed by global users via the open and public web. The web and the cloud resources are smartly combined and leveraged to ring in a series of delectable improvements and improvisations in the ways and means in which IT is being approached, analysed, and articulated. With the availability of slim and sleek devices, the cloud idea is to enter into a totally exotic and strange phase. One noteworthy derivative out of all these hot happenings in the cloud world is the eruption of service oriented cloud applications (SOCAs) due to the cool convergence of service orientation and cloud paradigms. The performance bottlenecks of SOCAs by taking into account a number of diverse scenarios can be observed. A new performance calculation as well as enhancement mechanism for this type of flexible and futuristic cloud-based applications can be presented [6, 12].

\section{SOA ARCHITECTURE}

SOA is a technology that makes it possible to link a variety of resources on a network for use as and when needed. The use of it, is more flexible in how resources are connected and utilized simultaneously than [8] in other types of system architecture designs. Service Oriented Architecture can be especially helpful when larger numbers of users are connected with the system, especially if there are remote users that must have access to specific applications residing on the network. SOA is an application architecture, in which application components or "services" are well defined using common interfaces, utilize a contract to define how services will be invoked, and interact in a loosely coupled manner.

\subsection{SOA LAYERS}

SOA Layers, where the business process, services and enterprise component's specific systems reside. The following diagram depicts the architecture of composite services which align with business processes.

Layer 1 - Operational Systems-It is the bottom layer which describes about the business operational systems, which contains legacy, non-legacy systems, ERP, CRM and old traditional systems.

Layer 2 - Component - It consists of technology specific business components, which provides the functional realization of services.

Layer 3 - Services - The Services layer consists of portfolio of service components specific to business units exposed as interfaces by means of service description (WSDL).

Layer 4 - Business Process - It provides the business orchestration of services which includes sequential flow of acts in a process. 
Layer 5 - Presentation- This Layer is out of scope from SOA prospective, but provides the means of communication to business through the channels like internet (WWW), mobile, ATM etc. Layer 6 -Integration- This layer is a back bone, which enables the integration of services through messaging, routing and transformation.

Layer $7-Q o S$ - This provides the security, performance and availability of service.

\begin{tabular}{|lc|}
\hline QoS, Security, Management and Monitoring & $\mathbf{7}$ \\
\hline $\begin{array}{l}\text { Integration Architecture } \\
\text { (Enterprise Service Bus) }\end{array}$ & $\mathbf{6}$ \\
\hline Presentation & $\mathbf{5}$ \\
\hline $\begin{array}{l}\text { Business Process } \\
\text { (Process choreography) }\end{array}$ & $\mathbf{4}$ \\
\hline $\begin{array}{l}\text { Services } \\
\text { (Composite Services) }\end{array}$ & $\mathbf{3}$ \\
\hline $\begin{array}{l}\text { Component-based } \\
\text { (Project or Enterprise Components) }\end{array}$ & $\mathbf{2}$ \\
\hline Operational Systems & $\mathbf{1}$ \\
\hline
\end{tabular}

Figure 1. SOA Layers

\subsection{WHY AND WHEN SOA?}

SOA is the existing technology which is implemented in industry. The technical characteristics are focused on the distributed technology and loosely coupled interfaces using open standards and driven through the process, message centric solutions. Web services are a common channel of integration across platforms in SOA which work on common communication transportation standards like XML, SOAP, and HTTP.

The following circumstances demand a need of SOA:

1. When there is a demand for high availability of business function.

2. When there is demand for Unified Information view.

3. When there is data not shared across channels.

4. Multiple applications need re-usable functions and data.

5. When there is increased operational cost.

6. When there is Business merger and acquisitions to expand their core business.

7. When there is increased business competition. And

8. Demand for more rapid business models, process across silos.

\subsection{WHEN NOT SOA?}

The following situations do not require a SOA solution: 
1. he business demands should be developed as simple as possible.

2. When the business cost does not reduce by operations by $10 \%$ or High.

3. When there is no improvement in customer satisfaction by $20 \%$.

4. The demand of the project is only to integrate the technologies.

5. The operational infrastructure must be as simple as possible.

6. When the service needs to provide a required unit of business functionality that supports business process and goals

7. The existence of the service help in eliminating redundant implementations, i.e. promote reuse.

\section{CORE INTEGRATION TECHNOLOGIES}

\subsection{ENTERPRISE SERVICE BUS}

Enterprise Service Bus is the channel for communication between parts, between applications which is accomplished by using messaging, transformation and routing. ESB providers provide adapters for interacting SOAP services, file systems, databases, FTP servers, Java Messaging Services, legacy systems, etc. Typically the services which are offered by ESB are messaging services, management services, Interface services, Mediation and Security services.

\subsection{Business Process Execution Language}

BPEL is a language for orchestrating web services to automate business process. It is evolved as the leading standard for web service integration and business process co-ordination. BPEL Follows three fundamental principles, asynchrony, flow co-ordination and exception management. Several Java Integrated Development Environments on the market offer extensive tool support for BPEL. These IDEs offer GUI based tools that allows the developer to drag and drop services and activities from a palette onto a graphical representation of the business process.

\subsection{BUSINESS RULES ENGINE}

Business rules engine separates the key deciding logic of a process. This is an abstraction layer which enables to change the rules faster and easier without affecting the business logic. The defined rules are available through a centralized syntactic layer, which are spread throughout the business process. BRE provides an ability to modify the execution of processes that will ultimately enhance the viability of each process solution.

\section{SYSTEM ARCHITECTURE BASED ON SOA CORE TECHNOLOGIES}

Following is the model system architecture of SOA using the core technologies. 


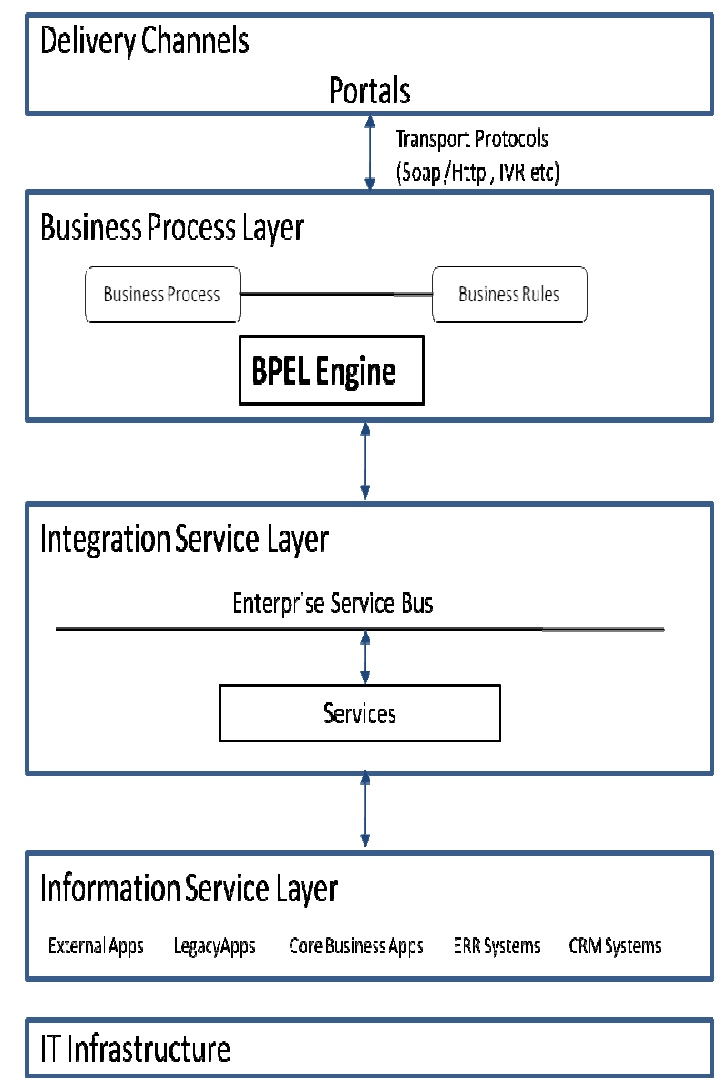

Figure 2. Model System Architecture of SOA

The model architecture comprises of Delivery channel Layer, Business Process Layer, Integration Service Layer and Information Solutions Layer.

A delivery channel provides a seamless integration with different enterprise application systems. Generally it provides services via portals, application programs, web service, ATM, Call Center etc.

Business Process Layer is mainly focused on orchestration to provide value to the respective business function both with respect to business and IT. Process is focused on organizing the service by connecting the coarse grained service to fine grained service for fulfilment of the business needs and routes to the respective service. This acts as an accelerator through the collaboration of undergoing business and service.

Integration Services Layer can be segregated as business service, information service, data service which connects to respective information resources for a particular business function. Usually the communication across the enterprise business information solutions is very demanding and difficult to re-use and co-ordinate in terms of functions and data. In SOA, messages are critical to deliver end-to-end services. Messages must be guaranteed a quick and correct delivery. To enhance messages transportation between services, we can use a core management service solution the enterprise service bus, which is the back bone of SOA architecture and helps in integrating with various enterprises business information solutions. ESB is a special layer that runs on top of the network that provides a guaranteed messaging service for most of the important messages on the network. This makes the Integration service layer and Information solutions layer playing a vital role in SOA. Ideally the business processes are isolated 
from business service, which makes these layers agile and helps in changing the business requirements and its process rapidly without having an impact on the other services.

\section{Quality of Service}

Mission critical enterprise systems require an essential and additional basic requirement of addressing the QoS requirements by adopting security, reliability and transactions. In general, security is one of the key environments, special concerns and considerations of every organization to protect business information, sensitive user data and establish trust relationships with organization business systems. As any business needs a flexible, customizable infrastructure, so that it can adapt to new requirements and regulations, as business needs a dynamic trust relationships with partners' customers and employees. To meet these business needs we need to leverage a security services infrastructure.

SOA environment should emphasize on some of the following key security challenges;

1. Need for user and service identities so that appropriate security controls are applied across the organization.

2. Need seamless connection to other organizations on real-time, transactional basis.

3. Need to manage identity and security across a range of systems and services that are implemented in a diverse mix of new and old technologies.

4. Need to protect the business data during transit and at rest.

5. Need for demonstrable compliance with a growing set of corporate, industry, and regulatory standards.

To address the above risks the main goals of SOA security are Confidentiality, Integrity, and Availability and in addition Authentication, Authorization, Auditing / Monitoring, Policy driven, and Hack proof. Authentication allows access only to the intended application that invokes the application. Authorization controls access to defined set of services and /or operations within a service. Auditing maintains a history of service calls and all activities within the SOA infrastructure. Integrity ensures that data which is entered is not corrupted. The Policy dictates the capability of the service provider by specifying web service's conditions under which the service is provided. Hack proof ensures that the service boundaries are not crossed to prevent several web service specific attacks such as XML Manipulation, schema attacks etc. The following are the industry standards for SOA Security which can be understood by every vendors and organizations that follow a common approach, so that the solutions are re-used, which benefit the parties by reducing the time, effort and investment and avoid them in re-inventing.

Figure 3. Industry Standards of SOA Security

\begin{tabular}{|l|l|}
\hline SOA Security & Standards \\
\hline Authentication & $\begin{array}{l}\text { WS-Security, WS-Trust, WS-Secure } \\
\text { Conversation }\end{array}$ \\
\hline Authorization & $\begin{array}{l}\text { XACML (eXtensible Access Control Mark-up } \\
\text { Language) }\end{array}$ \\
\hline Federation & SAML ( Single Sign-On) \\
\hline Policy & $\begin{array}{l}\text { WS-Policy, WS-Security Policy, WS-Metadata } \\
\text { Exchange }\end{array}$ \\
\hline Confidentiality & XML-Encrypt, SSL, XML-Signature \\
\hline Reliability & WS-Reliability, WS-Reliable Messaging \\
\hline
\end{tabular}


Enterprise Service Bus also allows the security and monitoring to be applied to services without modifying their core functionality. BAM (Business Activity Monitoring) is used to monitor the business process and end-to-end transactions.

\section{SOFTWARE PROVIDERS FOR SOA IMPLEMENTATION}

SOA platform is the technology component upon which organizations build, model, and implement the data models, services, and extensions. Because SOA services stretch across the enterprise, it is essential that the integration platform accommodate the organization's enterprisewide business processes. Finally, the vendor tools / software products would act as accelerators for integrating applications from one or multiple systems.

There are few software providers which respond to customer needs for agility, interoperability, business process and connectivity in their enterprise systems. Oracle is one of the software providers which fulfils the demands and needs of the organizations enterprise business, using SOA services.

The channel applications are the client applications which are either thick / thin or web based or mobile based applications developed in JEE / Microsoft / legacy Platform. These applications are very domain specific which are developed / customized for the organizations demands.

The following table provides the JEE environment software's for SOA Platform using Oracle Software Provider tools;

Figure 4. Sample Software Provider Solutions Using SOA

\begin{tabular}{|l|l|l|}
\hline Layer & Integration Technologies & Product Offerings \\
\hline Business Process Layer & BPM & Oracle BPA and BPEL \\
\cline { 2 - 3 } & Business rules & Oracle Business Rules \\
\hline $\begin{array}{l}\text { Integration Service } \\
\text { Layer }\end{array}$ & ESB & Oracle Service Bus \\
\hline QOS & $\begin{array}{l}\text { User Management ( } \\
\text { Security) }\end{array}$ & $\begin{array}{l}\text { Oracle Identity Management } \\
\text { (OID) }\end{array}$ \\
\cline { 2 - 3 } & Monitoring & $\begin{array}{l}\text { Oracle Business Activity } \\
\text { Monitoring ( BAM) }\end{array}$ \\
\cline { 3 - 3 } & Oracle WSM \\
\hline Operational Layer & Analytics & $\begin{array}{l}\text { Oracle Business Intelligence } \\
\text { (BI) }\end{array}$ \\
\hline
\end{tabular}

\section{ENTERPRISE BUSINESS INFORMATION SOLUTIONS REALIZATION USING SOA CORE TECHNOLOGIES}

In this section we have a discussion about realization of enterprise business information solutions using core technologies of SOA. Legacy systems are computer systems that have been in operation for a long time, and whose functions are too essential to be disrupted by upgrading or integration with another system despite its poor competitiveness. Legacy systems compatibility with modern equivalents has been facilitated via wrapper services. Wrapper service is a type of integration service that encapsulates and exposes logic of residing within a legacy system via standard Web services interface to be integrated in the new SOA based systems. Utilizing SOA, we can realize to build new applications within enterprises, which expose the ease of integration 
capabilities between newly adapted/developed applications and existing applications. The following figure 5 is an example of "Realization of Business Information Solutions Using SOA" The use case "New Credit Card Request" starts as follows, Existing end user requests for a "New Credit Card" online through the financial business portal. The submitted request by channel is sent as Soap over HTTP request which is processed by the SOA server. The location of the web service and WSDL information is sent to the application server. The request submitted by the user is executed asynchronously and the response will be sent to end user. The business information is processed by BPEL engine to execute the specific business process which is requested by the end user. The process executes sequential tasks to fulfil the requirement. As part of the process first the "User Information is captured from one of the business silo "SAP CRM", based on the user information, the external "Credit Score System" is connected by the service to decide whether the user is eligible for credit card or not. Next based on the user profile and credit score information the respective financial supervisor will approve or reject. After the approval, the request will be placed to create a credit card for the customer and his information will be maintained in the "Card Management System" which is a legacy system, which is hosted in Mainframe environment. Once the card is created the customer will be notified through the mail communication. The mail server is hosted as part of IT Infrastructure.

On realization of SOA system, it helps to adapt to business agility and respond to the speed of enterprise business.

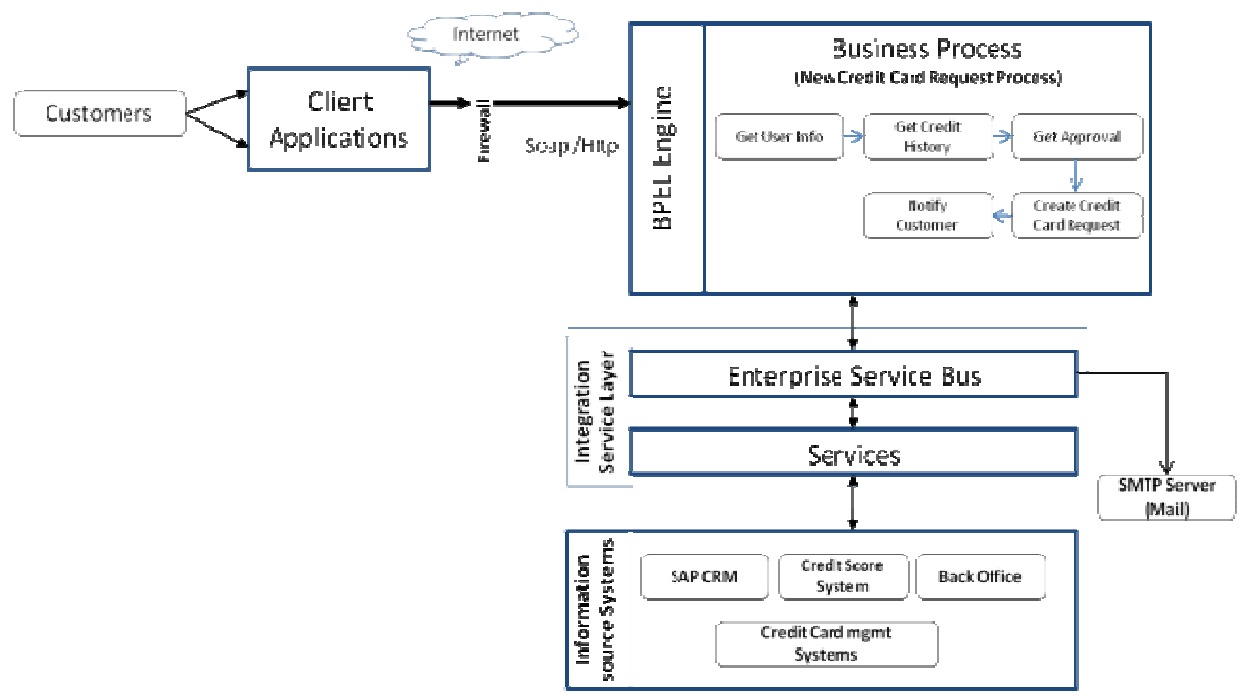

Figure 5. Realization of Business Information Solutions Using SOA

\section{CONCLuSion}

SOA is much more flexible, when compared to EAI (Enterprise Application Integration) and other architectures. It mainly focuses on the technological problem, system integration and service encapsulation, and BPM takes charge of the change of management mode. BPM requires enterprise, build definite process system and have effective management. The EAI based on SOA core technology can effectively improve the systems' response speed, and realize the complementary advantages of them. Therefore, from the aspects of the management and 
technology, the merging of enterprise information systems using SOA enhances the flexibility and responsiveness in the enterprise application systems and ultimately satisfies the business agility.

\section{REFERENCES}

[1] Jacqui Chetty, Marijke Coetzee, “Towards An Information Security Framework For Service-oriented Architecture", (C2010 IEEE

[2] Parichat Pasatcha, Komrhon Sunat, Mahanakorn University of Technology, Thailand," A Distributed e-Education System Based on the Service Oriented Architecture" 2008 IEEE International Conference on Web Services.

[3] Norman Ahmed, Mark Linderman, Jason Bryant, Rome, NY 13441, "Towards Mobile Data Streaming in Service Oriented Architecture”, 2010 29th IEEE International Symposium on Reliable Distributed Systems

[4] Zhao Yongyi, Feng Youjie, Liu Hui ,Shenyang, China, "Research on Service-Oriented Workflow Management System Architecture”, 2009 Ninth International Conference on Hybrid Intelligent Systems

[5] Alireza Hassanzadeh, Leila Namdarian, Tehran, Iran, "Developing a framework for service oriented architecture governance Maturity (SOAGM)", 2010 5th International Symposium on Telecommunications (IST'2010)

[6] Dr. R.Selvarani et.al. “ A Performance Analysis Method for Service-Oriented Cloud Applications (SOCAs)” 2012 IEEE International Conference on Computer Communication and Informatics (ICCCI 2012), Accepted December 2011

[7] Arya Amini, Soheila Sadjedy, Tehran, Iran, "SOMGIS- A Service Oriented Model for GIS Software Architecture", (2010 IEEE

[8] Poornachandra Sarang, Frank Jennings, Matjaz Juric, Ramesh Loganathan ,SOA Approach to Integration: XML, Web services, ESB, and BPEL in real-world SOA Dan Woods, Thomas Mattern,Enterprise SOA Design

[9] J. Loyall, M. Gillen, A. Paulos, L. Bunch, M. Carvallo, J. Edmondson, P. Varshneya, D. Schmidt and A. Martignoni III Dynamic Policy-Driven Quality of Service in Service-Oriented Systems, 13th IEEE Computer Society Symbosium, ISORC 2010

[10] SOA-based Multimedia Streaming Enabler over IMS Networks, In MoMM '09: Proceedings of the 7th International Conference on Advances in Mobile Computing and Multimedia (2009), pp. 391-395.

[11] Declan Dagger and Alexander O] Connor "Service-Oriented E-Learning P;atforms" IEEE Computer Society, 2007.

[12] Dr. R.Selvarani et al., "A New Performance Modelling and Measurement method for Service Oriented Cloud Applications" Fourth International Conference on Recent trends in Computing, communication and Information Technologies, Dec 9 - 11, 2011. Accepted September 2011.

[13] W. M. Omar and A. Taleb-Bendiab, "Service oriented architecture for e-health support services based on grid computing," Proceedings of the IEEE International Conference on Services Oriented Computing, Chicago, IL,September 2006, 135-142.

[14] A. Arsanjani, K. Holley, The service integration maturity model: Achieving flexibility in the transformation to SOA,IEEE International conference on services computing, 2006.

[15] S. Simanta, ED. Morris, G.A. Lewis, S. Balasubramaniam, D.B. smith, A scenario- based technique for developing SOA Technical Governance, software engineering institute, 2009.

[16] J. Rui-sheng1, J. Yan, S. Hong-mei, W. Xi-juan, Research on Distributed GIS Process Modeling and Integration, Proceedings of 2008 IEEE International Symposium on IT in Medicine and Education, 2008.

[17] D. Shujun, L. Liang, C. Chengq, Research on Geographical Information Service Based on SOA, Proceedings of the IEEE International Conference on Automation and Logistics Qingdao, China September, 2008. 
International Journal of Computer Science, Engineering and Applications (IJCSEA) Vol.2, No.2, April 2012

\section{ACKNOWLEDGEMENTS}

We are thankful for the Computer Science and Engineering department and management of M S Ramaiah Institute of Technology for having provided all the necessary research facilities.

\section{Biography:}

Dr R Selvarani: Dr R. Selvarani holds MI.Tech., Ph.D. in Computer Science and Engineering and has around 20 years of experience in teaching and research. She has published several research papers in computer science and holds two patents. She has been awarded Best Teacher Award twice in various institutions. Currently she is working as a Professor, Head of Department at M S Ramaiah Institute of technology, Bangalore, India.

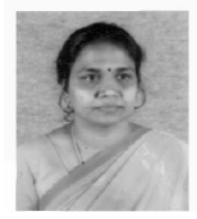

Rajashri Y Hiremath: I am pursuing my Mtech in Computer Science and Engineering at MS Ramaiah Institue of Technology, affiliated to Visvesvaraya Technological University, Bangalore, Karnataka. I worked in IT for around 5 years.

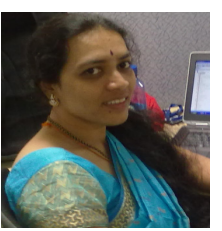

